\title{
Establishment of Quantitative Sequencing and Filter Contact Vial Bioassay for Monitoring Pyrethroid Resistance in the Common Bed Bug, Cimex lectularius
}

\author{
KEON MOOK SEONG, ${ }^{1}$ DA-YOUNG LEE,${ }^{2}$ KYONG SUP YOON ${ }^{3}$ DEOK HO KWON,${ }^{1}$ \\ HEUNG CHUL KIM, ${ }^{4}$ TERRY A. KLEIN, ${ }^{5}$ J. MARSHALL CLARK, ${ }^{3}$ \\ AND SI HYEOCK LEE LE $^{1,6}$
}

J. Med. Entomol. 47(4): 592-599 (2010); DOI: 10.1603/ME09274

\begin{abstract}
Two point mutations (V419L and L925I) in the voltage-sensitive sodium channel $\alpha$-subunit gene have been identified in deltamethrin-resistant bed bugs. A quantitative sequencing (QS) protocol was developed to establish a population-based genotyping method as a molecular resistance-monitoring tool based on the frequency of the two mutations. The nucleotide signal ratio at each mutation site was generated from sequencing chromatograms and plotted against the corresponding resistance allele frequency. Frequency prediction equations were generated from the plots by linear regression, and the signal ratios were shown to highly correlate with resistance allele frequencies $\left(r^{2}>0.9928\right)$. As determined by QS, neither mutation was found in a bed bug population collected in 1993. Populations collected in recent years (2007-2009), however, exhibited completely or nearly saturating L925I mutation frequencies and highly variable frequencies of the V419L mutation. In addition to QS, the filter contact vial bioassay (FCVB) method was established and used to determine the baseline susceptibility and resistance of bed bugs to deltamethrin and $\lambda$-cyhalothrin. A pyrethroid-resistant strain showed $>9,375$ - and 6,990-fold resistance to deltamethrin and $\lambda$-cyhalothrin, respectively. Resistance allele frequencies in different bed bug populations predicted by QS correlated well with the FCVB results, confirming the roles of the two mutations in pyrethroid resistance. Taken together, employment of QS in conjunction with FCVB should greatly facilitate the detection and monitoring of pyrethroid-resistant bed bugs in the field. The advantages of FCVB as an on-site resistance-monitoring tool are discussed.
\end{abstract}

KEY WORDS Cimex lectularius, pyrethroid resistance, sodium channel mutation, quantitative sequencing, filter paper contact vial bioassay

The common bed bug, Cimex lectularius L. (Hemiptera: Cimicidae), is an ectoparasitic pest that feeds on humans as well as other mammals (Usinger 1966, Day et al. 2000, Reinhardt and Siva-Jothy 2007). It is widely distributed in temperate and subtropical regions and is considered a serious indoor nuisance (Ter Poorten and Prose 2005). Although bed bugs are not considered a disease vector, they can mechanically transmit hepatitis B virus (Blow et al. 2001) and human immunodeficiency virus (Jupp and Lyons 1987).

\footnotetext{
${ }^{1}$ Department of Agricultural Biotechnology, Seoul National University, Seoul 151-921, Republic of Korea.

2 Amherst Regional High School, Amherst, MA 01002.

${ }^{3}$ Department of Veterinary and Animal Sciences, University of Massachusetts, Amherst, MA 01003.

${ }^{4}$ Fifth Medical Detachment, 168th Multifunctional Medical Battalion, 65th Medical Brigade, Unit 15247, APO AP 96205-5247.

${ }^{5}$ Force Health Protection and Preventive Medicine, 65th Medical Brigade/USA MEDDAC-Korea, Unit 15281, APO AP 96205-5281.

${ }^{6}$ Corresponding author: Department of Agricultural Biotechnology, Seoul National University, Seoul, Korea 151-742 (e-mail: shlee22@snu.ac.kr).
}

Introduction of synthetic insecticides after World War II greatly reduced bed bug populations with very low rate of infestation until the beginning of this century (Scarupa and Economides 2006). In recent years, however, the incidence of bed bug infestation has increased dramatically in the United States and in the United Kingdom (Ter Poorten and Prose 2005). Increased levels of international travel, trade, and immigration have undoubtedly contributed to the prevalence of this household pest (Ter Poorten and Prose 2005). Currently, the application of insecticides is the only effective means to control bed bugs. Natural pyrethrins and synthetic pyrethroids, including deltamethrin, cyhalothrin, and permethrin, have been used extensively for bed bug control in residences, hotels, and commercial operations (Gangloff-Kaufmann et al. 2006).

Unfortunately, the extensive use of this class of insecticides has resulted in resistance in bed bug populations. Pyrethroid and carbamate resistance in bed bugs was identified in a field-collected population in the United Kingdom (Boase 2001). Recently, bed bug 
Table 1. PCR and sequencing primers used in this study

\begin{tabular}{|c|c|c|}
\hline Primer name & Sequence & Remarks \\
\hline $5^{\prime} \mathrm{BB}-\mathrm{QS}(\mathrm{VL})$ & 5'-GTCCGTGGCACATGTTGTTCTTCA-3' & For the cloning of partial Clvssc \\
\hline $3^{\prime} \mathrm{BB}-\mathrm{QS}(\mathrm{VL})$ & 5'-CTGATGGAGATTTTGCCACTGATGC-3' & fragments \\
\hline $5^{\prime} \mathrm{BB}-\mathrm{QS}(\mathrm{LI})$ & 5'-GGTCTATCAGTTTTGAGGTCATTCAG-3' & \\
\hline $3^{\prime} \mathrm{BB}-\mathrm{QS}(\mathrm{LI})$ & 5'-GGAGTTCGCCATCAGGGAATCTAT-3' & \\
\hline 3'QS-VL & 5'-CCTCTTCAGCAGCTTCTTCTTCTTC-3' & For sequencing \\
\hline $5^{\prime} \mathrm{QS}-\mathrm{LI}$ & 5'-GTGTTTAAGCTGGCTAAGTCATGGCC-3' & \\
\hline 3'QS-LI & 5'-CCAAACAACTGCATTCCCATCACAGC-3' & \\
\hline
\end{tabular}

populations that are resistant to deltamethrin and $\lambda$-cyhalothrin have been reported to exist in the United States (Moore and Miller 2006, Romero et al. 2007). Molecular analysis of deltamethrin resistance in a bed bug population collected from New York City indicated that two point mutations (V419L and L925I) in the voltage-sensitive sodium channel (VSSC) $\alpha$-subunit were primarily responsible for nerve insensitivity, resulting in knockdown resistance $(k d r)$, whereas metabolic factors did not seem to be involved at this early stage of resistance (Yoon et al. 2008).

Efficient detection of resistance at an early stage of development is a crucial factor in resistance management. An insecticide-impregnated paper method (IIPM) was initially used to monitor insecticide resistance in the tropical bed bug, Cimex hemipterus (Karunaratne et al. 2007). A modified version of IIPM using a smaller filter paper disk ( $52 \mathrm{~mm}$ diameter) was used for evaluating bed bug resistance to deltamethrin (Yoon et al. 2008). These bioassay methods can be employed for resistance monitoring of field populations of bed bugs when a sufficient number of live specimens are available. Because obtaining a large number of live bed bugs for bioassays is difficult, routine monitoring of bed bug resistance based on these bioassay methods is often unfeasible. Moreover, resistance monitoring by bioassay can be compromised when the resistance allele is recessive and a large number of heterozygotes are present in the insect population. Therefore, alternative monitoring protocols based on molecular techniques to complement bioassay-based resistance monitoring are a critical need in the long-term resistance management of bed bugs.

In this study, a quantitative sequencing (QS) protocol was established to predict the allelic frequencies of the two mutations in the bed bug VSSC $\alpha$-subunit gene (Clvssc) that are associated with pyrethroid resistance. This population-based genotyping method can be employed as a routine resistance-monitoring tool. In addition, a new bioassay method modified from IIPM was developed, and its usefulness for onsite resistance detection was discussed. Finally, the function of the two mutations in resistance was evaluated by correlating actual resistance levels to Clvssc mutation allele frequencies.

\section{Materials and Methods}

Bed Bug Populations. Insecticide-susceptible (FLBB) and pyrethroid-resistant (NY-BB) bed bug pop- ulations were obtained and maintained, as described previously (Yoon et al. 2008). Because the original NY-BB strain showed apparent symptoms of fitness disadvantage after consecutive inbreeding, such as reduced fecundity and increased development time, it was outbred with the FL-BB strain by randomly mixing an equal number of adults from each strain. The mixed population (NYM-BB) was maintained for $\approx 10$ mo and then selected with deltamethrin $(>70 \%$ mortality) using a modified IIPM method (Yoon et al. 2008) for two consecutive generations to obtain a highly resistant strain (NYS-BB).

Determination of Exon-Intron Structure in a Partial Genomic DNA Fragment of Clvssc. Genomic DNA was extracted using the DNeasy tissue kit (Qiagen, Valencia, CA), according to the manufacturer's instructions. Briefly 10 bed bugs (FL-BB or NY-BB) were homogenized with $180 \mu \mathrm{l}$ of ATL buffer (Qiagen) and treated with proteinase $\mathrm{K}$ at $55^{\circ} \mathrm{C}$ for $3 \mathrm{~h}$. The homogenate was mixed with $200 \mu \mathrm{l}$ of AL buffer (Qiagen) and $200 \mu \mathrm{l}$ of $96 \%$ ethanol. The mixture was centrifuged through the supplied spin column, and genomic DNA was eluted with $100 \mu$ l Tris-EDTA buffer. Two DNA fragments of Clvssc, encompassing the V419L or L925I mutation site, were polymerase chain reaction (PCR) amplified from individual genomic DNA templates from either the NY-BB or FL-BB strains using sequence-specific primer pairs (Table 1). PCR thermal cycling was performed for 35 cycles of $95^{\circ} \mathrm{C}$ for $30 \mathrm{~s}, 61^{\circ} \mathrm{C}$ for $30 \mathrm{~s}, 72^{\circ} \mathrm{C}$ for $1 \mathrm{~min}$, and one cycle of $72^{\circ} \mathrm{C}$ for $10 \mathrm{~min}$. The PCR products were directly subcloned into the pGEMTeasy vector (Promega, Madison, WI) and transformed into One Shot TOP10 chemically competent Escherichia coli (Invitrogen, Carlsbad, CA). Positive clones were identified by colony PCR using supplied universal primer sets. A total of 10 positive clones was sequenced to determine the exon-intron structure and the sequence variations between clones with an ABI Prism 3730 DNA sequence analyzer (NICEM Sequencing Facility, Seoul National University, Seoul, Korea).

Comparison of Genotype Versus Transcript Type at the Two Mutation Sites. Both total RNA and genomic DNA were extracted from the same individual bed bugs with known genotypes (homozygous susceptible and resistant versus heterozygous) at the two mutation sites of Clvssc using TRI reagent (MRC, Cincinnati, $\mathrm{OH}$ ). Briefly, $100 \mu \mathrm{l}$ of TRI reagent was used to homogenize a single bed bug, and subsequent procedures were based on the manufacturer's instructions. cDNA was synthesized from total RNA (1.5 $\mu \mathrm{g})$ using 
Superscript III reverse transcriptase (Invitrogen, Carlsbad, CA) by priming with oligo(dT). PCRs using genomic DNA (30 ng) or cDNA (15 ng) templates were conducted to amplify the genomic or cDNA fragments, respectively, of Clvssc flanking the two mutation sites following the same method described in the previous section. The PCR products from both genomic DNA and cDNA of a single individual were purified using a QIAquick PCR purification kit (Qiagen, Valencia, CA) and directly sequenced, as described above, to determine the genotypes or transcript types at each mutation site.

Establishment of the QS Protocol for Detection of Resistance Mutations. A QS protocol was developed according to the method of Kwon et al. (2008) with some modification. The genomic DNA fragment of Clvssc encompassing either the V419L or L925I mutation site was PCR amplified from individual genomic DNA templates extracted from either the NY-BB or FL-BB strains, as described above. The PCR products were purified using a QIAquick PCR purification kit (Qiagen, Valencia, CA) and directly sequenced as above to confirm the genotypes at each mutation site. Once the genotype was identified, the purified PCR products were quantified using a NanoDrop ND-1000 spectrophotometer (NanoDrop Technologies, Wilmington, DE) and used as standard DNA templates. The standard DNA templates were mixed in the following molar ratios: $0: 10,1: 9,2: 8,3: 7,5: 5$, and $7: 3$ (resistant allele:susceptible allele at each mutation site). Standard DNA template mixtures (20 ng) were sequenced using a sequencing primer (antisense primer for V419L versus sense primer for L925I) (Table 1). The nucleotide signal intensities of the resistant and susceptible alleles at each mutation site were measured from the sequence chromatogram using Chromas version 2.31 software (Technelysium, Tewantin, Australia), and the signal ratios (resistant nucleotide signal/ [resistant nucleotide signal + susceptible nucleotide signal]) were calculated. Standard linear regression equations were generated from the plots of the resistance allele frequencies versus corresponding nucleotide signal ratios using the SIGMA plot version 10.0 (Systat Software, San Jose, CA) and used to predict resistance allele frequencies of unknown samples. Likewise, the lower and upper prediction equations were generated to estimate their prediction intervals at the $95 \%$ confidence level.

Filter Contact Vial Bioassay (FCVB). Technical grade deltamethrin and $\lambda$-cyhalothrin ( $>99 \%$ pure, Chem Service, West Chester, PA) were dissolved in acetone to appropriate concentrations. A filter paper (Whatman No. 1) strip $(13 \times 35 \mathrm{~mm})$ was dipped into an appropriate concentration of pyrethroid for $10 \mathrm{~s}$ and dried in a darkened fume hood for $30 \mathrm{~min}$. Bed bugs (third instar) were collected using a custommade aspirator fitted with a 5-ml glass vial (Taeshin Bioscience, Guri, Korea). The pyrethroid-treated filter paper strip was longitudinally placed into a 5-ml glass vial containing 10 bed bugs. Control filter paper strips were treated with acetone only. The vials were tightly capped and kept erect during mortality assess- ment. All bed bugs clung to the filter paper strip because they were unable to crawl over the smooth inner surface of the glass vial. Bed bugs that fell from the filter strip and remained immobile on the bottom of vial were considered dead. The $\mathrm{LC}_{50}$ and $\mathrm{LC}_{99}$ values were determined by Probit analysis (POLO PC, LeOra Software, Berkeley, CA). All tests were performed at $28 \pm 1^{\circ} \mathrm{C}$ and repeated at least three times on different days.

Correlation Between Clvssc Mutation Frequencies and $\boldsymbol{\lambda}$-Cyhalothrin Resistance Levels. To investigate the correlation between Clvssc mutation frequencies and resistance levels of bed bugs to $\lambda$-cyhalothrin, frequencies of V419L or L925I mutation were evaluated by QS and the $\mathrm{LC}_{50}$ values were determined by FCVB for three bed bug strains (FL-BB, NYM-BB, and NYS-BB) that differed in their resistance levels to $\lambda$-cyhalothrin. The frequencies of V419L or L925I mutation were plotted against $\mathrm{LC}_{50}$ values, and correlation equations were determined using SIGMA plot version 10.0 (Systat Software, San Jose, CA).

Evaluation of Resistance Allele Frequencies in Field-Collected Bed Bugs. Nymph and adult bed bugs were collected from several locations within the U.S. Army Garrison, Yongsan, Seoul, Korea in 1993, 2007, 2008 , and 2009, and stored in $95 \%$ ethyl alcohol at $-20^{\circ} \mathrm{C}$. Genomic DNA was extracted from 10 third instars. The 354-bp DNA fragment containing the V419L mutation site and the 360-bp fragment containing the L925I mutation site were amplified to predict resistance allele frequencies by QS, as described previously. Resistance allele frequencies were analyzed using standard regression equations.

\section{Results}

Exon-Intron Structure of Clvssc Genomic DNA Fragments Encompassing the V419L and L925I Mutation Sites. Two separate genomic DNA fragments of Clvssc (354 and 360 bp containing the V419L and L925I mutation sites, respectively) were PCR amplified, and their sequences were determined. Their exon-intron structures are illustrated in Fig. 1. The 354-bp fragment covering the IS5-IS6 segment is composed of two exons (150 and $140 \mathrm{bp}$ ), with the V419L mutation occurring in exon 1 . The 360-bp fragment encompassing the IIS4-IIS5 segment is composed of three exons, with the L925I mutation located in exon 2 (160 bp). Sequencing of 10 individual clones of each fragment confirmed that no sequence variation exists within the exonic regions except for the V419L and L925I mutations. In contrast, some sequence variations were observed within the intron regions. Therefore, the sequencing primers for QS were designed from sequences within the same exon that contained the mutation site of interest.

Genotype Versus Transcript Type at the Two Mutation Sites. A total of 15 individual bed bugs (five homozygous susceptible, five homozygous resistant, and five heterozygous) was analyzed. Representative chromatograms for each genotype versus transcript type are shown in Fig. 2. The transcript type of each 
(A) 354 bp genomic DNA fragment

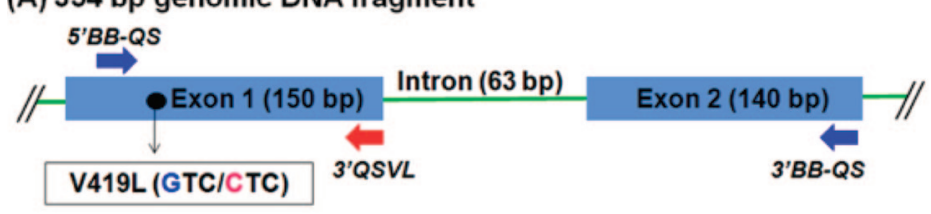

(B) 360 bp genomic DNA fragment

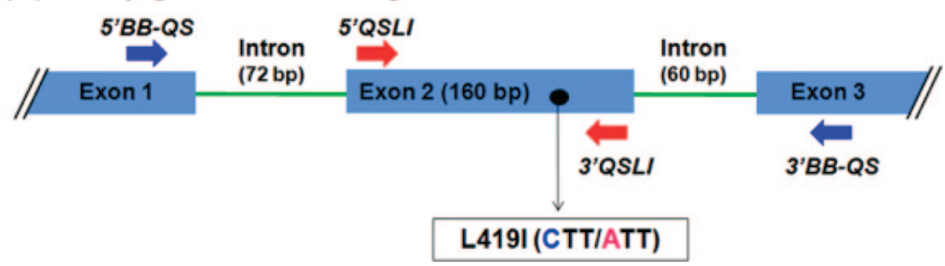

Fig. 1. Exon-intron structure of the 354- and 360-bp genomic fragments of Clvssc, encompassing the V419L (A) and L925I (B) mutation sites, respectively. Shaded boxes and solid lines indicate exons and introns, respectively. Locations of two resistance mutations are marked with $\bullet$. Horizontal arrows indicate the locations of the QS primers. (Online figure in color.)

individual was identical to its genotype without exception.

Establishment of the QS Protocol to Predict Resistance Allele Frequency. When a set of standard DNA template mixtures, which were adjusted to contain an increasing frequency of the resistant allele, was sequenced, the resistant nucleotide signal intensity increased according to the resistance allele frequency in the template DNA mixtures (Fig. 3). The nucleotide signal ratios were plotted against the corresponding resistance allele frequencies at each mutation site and fitted to linear equations (Fig. 4). The two resulting regression lines both revealed high correlation coefficients $\left(r^{2}=0.9928\right.$ and 0.9997$)$, demonstrating that the nucleotide signal ratios are highly proportional to the resistance allele frequencies. The prediction equations generated from antisense directional sequencing of the V419L mutation resulted in relatively wider $95 \%$ prediction intervals (Fig. 4A), whereas sequencing of the L925I mutation in the sense direction generated considerably narrower intervals (Fig. 4B). Using the lower and upper $95 \%$ prediction equations, the lower detection limit for the V419L mutation allele was calculated to be $6.1 \%$ at the $95 \%$ confidence level. Likewise, the detection limit for the L925I mutation allele frequency was determined to be $2.0 \%$ at the $95 \%$ confidence level.

Establishment of the FCVB Protocol. A series of increasing concentrations of deltamethrin or $\lambda$-cyhalothrin were applied to both FL-BB and NYS-BB using the FCVB method to determine baseline susceptibility and resistance levels. When mortality was evaluated $12 \mathrm{~h}$ posttreatment, resistance levels of NYS-BB to deltamethrin and $\lambda$-cyhalothrin were $>9,375$ - and 6,990-fold higher than FL-BB, respectively, as determined by $\mathrm{LC}_{50}$ values (Table 2 ). Precise estimation of the deltamethrin $\mathrm{LC}_{50}$ for the NYS-BB strain was not feasible because its mortality, even at the highest concentration examined $(30,000 \mathrm{ppm})$, was still $<50 \%$. Apparently cross-resistance exists between the two

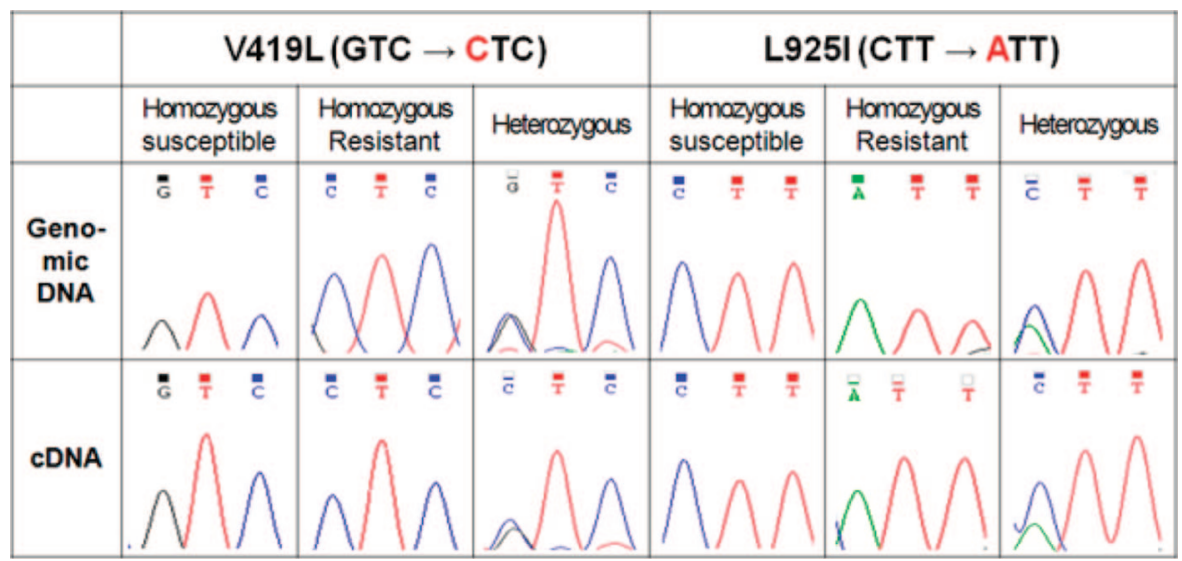

Fig. 2. Comparison of genotype (genomic DNA) and transcript type (cDNA) at the V419L and L925I mutation sites of Clvssc. Typical sequencing chromatograms from bed bugs with the three different genotypes (homozygous susceptible, homozygous resistant, and heterozygous) are shown. (Online figure in color.) 


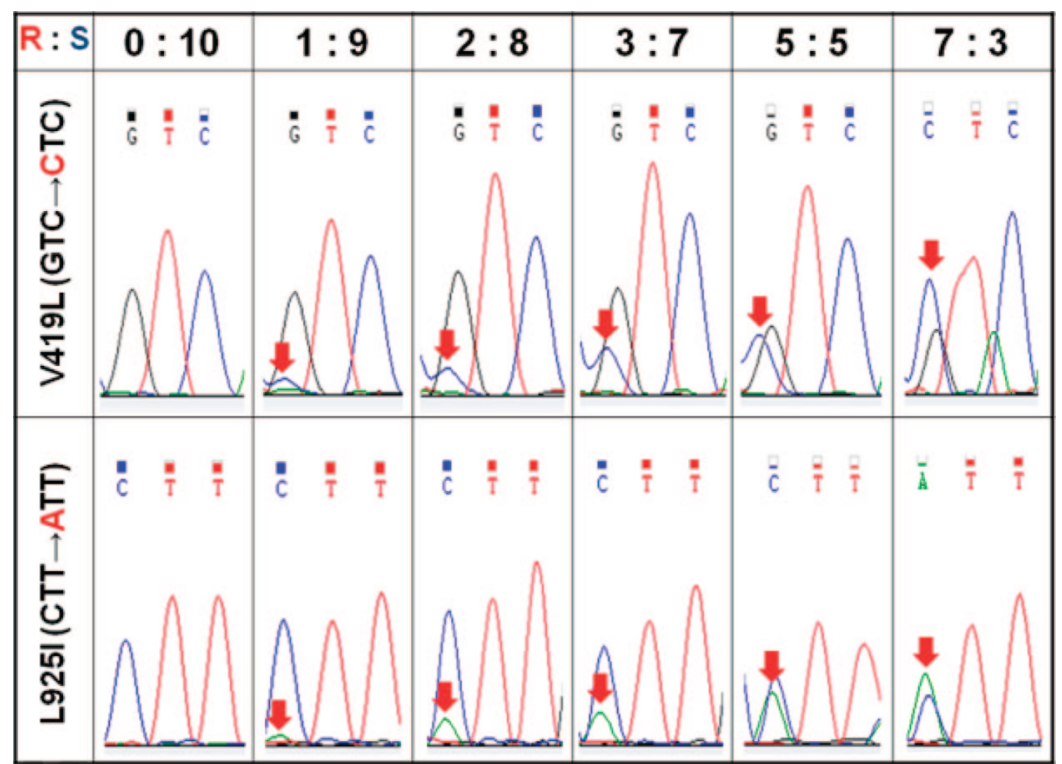

Fig. 3. Sequencing chromatograms of the standard template DNA mixtures with different ratios of resistant and susceptible alleles at the V419L and L925I mutation sites. The numbers at the top of each column indicate the resistance allele versus the susceptible allele frequencies at each mutation site. The relative intensities of the resistant nucleotide signals are indicated with arrows. (Online figure in color.)

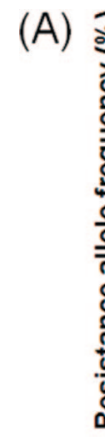

(B)
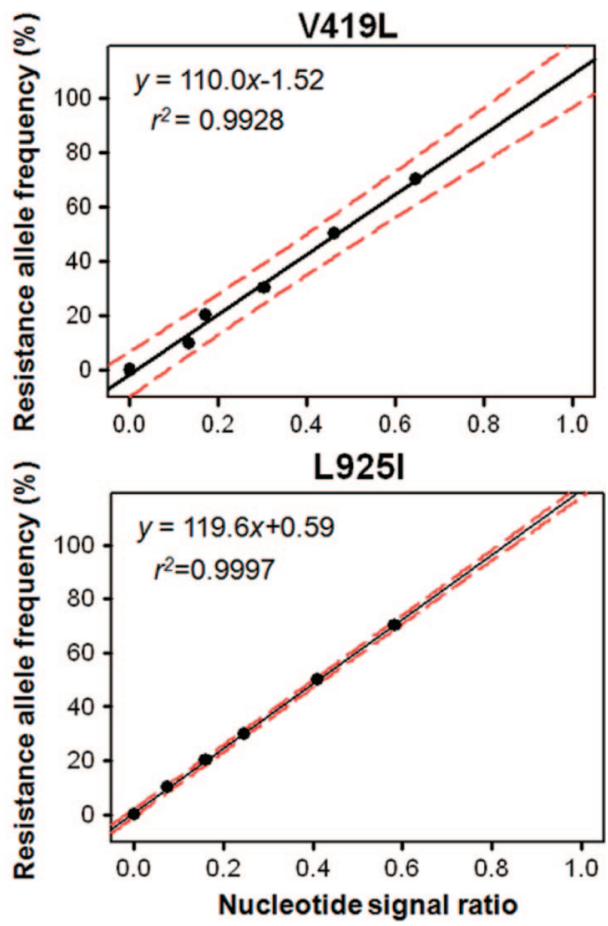

Fig. 4. Resistance nucleotide signal ratios obtained from sequencing were plotted against corresponding resistance allele frequencies at the V419L (A) and L925I (B) mutation sites. Linear regression lines are indicated by solid black lines with the upper and lower $95 \%$ prediction lines demarcated by dotted red lines. The nucleotide signal ratio ( $x$-axis) was calculated as follows: resistant nucleotide signal/ [resistant nucleotide signal + susceptible nucleotide signal]. (Online figure in color.) pyrethroids in that the responses of NYS-BB to deltamethrin and $\lambda$-cyhalothrin were similar. The concentrations capable of killing most susceptible bed bugs $\left(\mathrm{LC}_{99}\right) 12 \mathrm{~h}$ posttreatment were estimated to be 26 and $30 \mathrm{ppm}$ for deltamethrin and $\lambda$-cyhalothrin, respectively. When evaluated at $1 \mathrm{~h}$ posttreatment, the $\mathrm{LC}_{99}$ concentrations were approximately 3 -fold higher for both pyrethroids. No NYS-BB bed bugs were killed by even the highest concentrations of deltamethrin or $\lambda$-cyhalothrin tested $(30,000 \mathrm{ppm})$ within $1 \mathrm{~h}$ posttreatment. Using a concentration 2 -fold greater than the $\mathrm{LC}_{99}$ value as the threshold to ensure $100 \%$ mortality of pyrethroid-susceptible bed bugs, the discriminating concentrations for deltamethrin and $\lambda$-cyhalothrin were estimated to be 54 and $60 \mathrm{ppm}$ at $12 \mathrm{~h}$ posttreatment and 164 and $174 \mathrm{ppm}$ at $1 \mathrm{~h}$ posttreatment, respectively.

Correlation of the Frequency of the Two Clvssc Mutations to Pyrethroid Resistance in LaboratorySelected Bed Bug Strains. The $\lambda$-cyhalothrin $\mathrm{LC}_{50}$ for NYM-BB strain, measured $\approx 10$ mo after outbreeding to the FL-BB strain, was $62 \mathrm{ppm}$. After a single round of selection of the NYM-BB strain with deltamethrin, the $\lambda$-cyhalothrin $\mathrm{LC}_{50}$ of the resulting selected strain (NYMS-BB) increased to $16,917 \mathrm{ppm}$. The NYS-BB strain was established after one additional selection with deltamethrin. The $\lambda$-cyhalothrin LC $_{50}$ for NYS-BB strain was estimated to be $23,765 \mathrm{ppm}$, which was a 383-fold increase in resistance over its progenitor population, NYM-BB.

The frequencies of the V419L mutation in the FLBB, NYM-BB, NYMS-BB, and NYS-BB strains were determined to be $0,44,91$, and $100 \%$, respectively. Likewise, the frequencies of the L925I mutation grad- 
Table 2. Evaluation of lethal effects of deltamethrin or $\lambda$-cyhalothrin on pyrethroid-resistant (NYS-BB) and -susceptible (FL-BB) strains of bed bugs using the FCVB

\begin{tabular}{|c|c|c|c|c|c|c|c|c|c|c|c|}
\hline \multirow{2}{*}{ Insecticide } & \multirow{2}{*}{$\begin{array}{c}\text { Bed } \\
\text { bug strain }\end{array}$} & \multirow{2}{*}{$n$} & \multicolumn{2}{|c|}{ Slope \pm SE } & \multicolumn{2}{|c|}{$\mathrm{LC}_{50}\left(95 \% \mathrm{CI}^{a}\right), \mathrm{ppm}$} & \multicolumn{2}{|c|}{$\mathrm{LC}_{99}(95 \% \mathrm{CI}), \mathrm{ppm}$} & \multicolumn{2}{|c|}{$\chi^{2}$} & \multirow{2}{*}{$\mathbf{R R}^{b}$} \\
\hline & & & $1 \mathrm{~h}$ & $12 \mathrm{~h}$ & $1 \mathrm{~h}$ & $12 \mathrm{~h}$ & $1 \mathrm{~h}$ & $12 \mathrm{~h}$ & $1 \mathrm{~h}$ & $12 \mathrm{~h}$ & \\
\hline \multirow[t]{2}{*}{ Deltamethrin } & FL-BB & 150 & $3.4 \pm 0.5$ & $2.6 \pm 0.4$ & $18.1(13.6-22.9)$ & $3.2(2.5-4.0)$ & $81.8(53.7-233)$ & $26.0(15.1-73.2)$ & 10.4 & 3.6 & $>9,375$ \\
\hline & NYS-BB & 150 & - & $1.5 \pm 0.4$ & - & $>30,000$ & - & $>30,000$ & - & 4.2 & \\
\hline \multirow[t]{2}{*}{$\lambda$-Cyhalothrin } & FL-BB & 150 & $3.3 \pm 0.7$ & $2.5 \pm 0.4$ & $17.7(14.2-22.9)$ & $3.4(2.7-4.4)$ & $87.0(60.1-263)$ & $30.0(17.0-90.4)$ & 2.4 & 7.8 & 6,990 \\
\hline & NYS-BB & 150 & - & $1.5 \pm 0.4$ & - & $23,765(16,062-54,008)$ & - & $770,103(189,318-45,701,321)$ & - & 3.3 & \\
\hline
\end{tabular}

${ }^{a}$ Confidence interval.

${ }^{b}$ Resistance ratio $=\mathrm{LC}_{50}$ of NYS-BB $/ \mathrm{LC}_{50}$ of FL-BB.

ually increased as selection proceeded $(0,37,85$, and $100 \%$ in the FL-BB, NYM-BB, NYMS-BB, and NYS-BB strains, respectively). When the frequencies of the V419L or L925I mutation were plotted against the $\mathrm{LC}_{50}$ values (Fig. 5), they significantly correlated with resistance levels, as indicated by high correlation coefficients $\left(r^{2}=0.9820\right.$ for V419L frequency and 0.9854 for L925I frequency). As the frequency of Clvssc mutations in bed bug strains increased, the actual level of resistance to deltamethrin increased. This finding is consistent with a role of the two mutations in pyrethroid resistance.

Frequencies of Clvssc Mutations in Field-Collected Bed Bug Specimens. Frequencies of the two Clvssc mutations in bed bug populations collected from different locations and times from the Yongsan area were

(A)

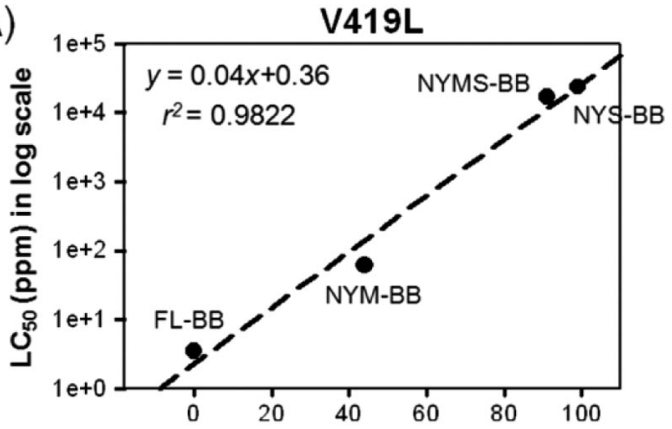

(B)

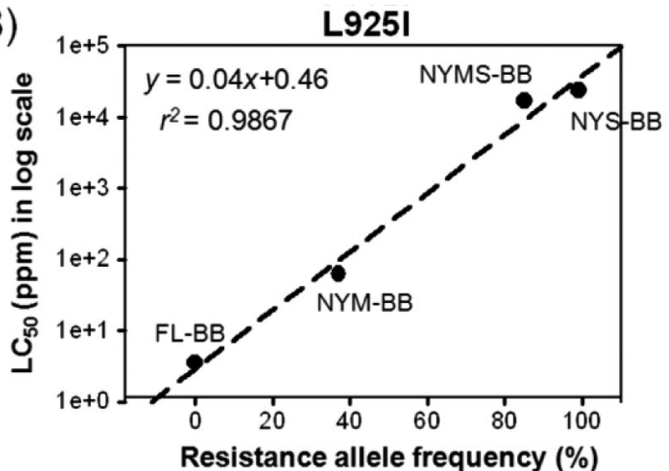

Fig. 5. Resistance allele frequencies of the V419L (A) and L925I (B) mutations in four bed bug strains (FL-BB, NYM-BB, NYMS-BB, and NYS-BB) were plotted against corresponding $L_{50}$ values. Linear regression lines are indicated by dashed black lines. Resistance allele frequencies ( $x$-axis) were calculated by QS. predicted by QS (Table 3). No mutations were found in the population collected in 1993 (Yongsan A). By 2007, however, the frequencies of both the V419L and L925I mutations were $100 \%$ (Yongsan B). Interestingly, the frequency of the V419L mutation (8\%) was much lower than that of L925I mutation (86\%) in the bed bugs collected in 2008 (Yonsan C). This trend of segregation between the two mutations became more apparent in bed bugs collected in 2009 (Yongsan D), with $0 \%$ carrying the V419L mutation and $100 \%$ carrying the L925I mutation. Based on these findings, it was apparent that there are at least two different Clvssc haplotypes in terms of mutations (one having only the L925I mutation, and the other having both V419L and L925I mutations). All bed bug populations collected in more recent years (2007-2009) appeared to have saturated or nearly saturating frequencies of L925I mutation. However, the V419L mutation frequency was highly variable in its frequency, suggesting that it is not yet uniform.

\section{Discussion}

The role of the two Clvssc mutations, V419L and L925I, in pyrethroid-resistant bed bugs was strongly supported by the fact that the frequencies of both mutations were highly proportional to the level of resistance (Fig. 5). The correlation coefficient of the L925I mutation $\left(r^{2}=0.9854\right)$ was similar to that of the V419L mutation $\left(r^{2}=0.9820\right)$, suggesting that the

Table 3. Clvssc mutation frequencies in field-collected bed bugs predicted by QS

\begin{tabular}{|c|c|c|c|c|}
\hline \multirow{2}{*}{$\begin{array}{c}\text { Bed bug } \\
\text { population/ } \\
\text { collection time }\end{array}$} & \multicolumn{2}{|r|}{ V419L } & \multicolumn{2}{|r|}{ L925I } \\
\hline & $\mathrm{SR}^{a}$ & $\begin{array}{l}\text { Frequency } b \\
(95 \% \mathrm{PI}),{ }^{c} \%\end{array}$ & $\mathrm{SR}^{a}$ & $\begin{array}{l}\text { Frequency } b \\
(95 \% \text { PI }),{ }^{c} \%\end{array}$ \\
\hline $\begin{array}{l}\text { Yongsan A/Sept. } \\
1993\end{array}$ & 0 & 0 & 0 & 0 \\
\hline $\begin{array}{l}\text { Yongsan B/June } \\
\quad 2007\end{array}$ & 1.0 & 100 & 1.0 & 100 \\
\hline $\begin{array}{l}\text { Yongsan C/Aug. } \\
2008\end{array}$ & 0.09 & $8.0(0.7-16.1)$ & 0.72 & $86(85.1-92.4)$ \\
\hline $\begin{array}{l}\text { Yongsan D/May } \\
2009\end{array}$ & 0 & 0 & 1.0 & 100 \\
\hline
\end{tabular}

${ }^{a}$ Signal ratio $=$ resistant nucleotide signal $/$ (resistant nucleotide signal + susceptible nucleotide signal).

${ }^{b}$ If the signal ratio is 0 or 1.0 , the resulting mutation frequency was considered to be 0 or $100 \%$, respectively, without incorporating it into the prediction equation.

${ }^{c} 95 \%$ prediction interval. 
function of each mutation in conferring resistance is almost identical. The genotypes of the two Clvssc mutations matched completely with transcript types, suggesting that RNA editing does not occur in bed bugs, unlike in Culex quinquefasciatus mosquitoes, in which the alteration of transcript by RNA editing results in resistance (Xu et al. 2006). Because RNA editing is not involved in the expression of the pyrethroid resistance allele in bed bugs, genotyping of the Clvssc mutations can be reliably used to evaluate the presence or absence of the pyrethroid resistance mediated by the knockdown resistance $(k d r)$ factor(s).

A QS protocol was developed and used for the prediction of Clvssc mutation frequencies as an efficient and cost-effective genotyping tool for the detection of pyrethroid resistance in field-collected bed bug populations. The lower detection limits of QS were 6.1 and 2.0\% for the V419L and L925I mutations, respectively. To guarantee reliable detection at the lower limit, a minimum number of $\approx 16-50$ bed bugs, depending on the mutation site, would be required for the analysis of a field population. A QS analysis with this number of bed bug specimens (for example, 16-50 bed bugs per population) can be conducted cost effectively. Moreover, its simplicity and accuracy make QS an ideal choice for use as an initial routine resistance-monitoring tool to screen a large number of wild bed bug populations as an alternative to conventional bioassays (Lee et al. 2009, Clark 2009). If more precise determination of resistance allele frequency below the QS detection limit is required on a population basis, real-time PCR amplification of specific allele (Kwon et al. 2004) can be employed as a secondary or validating resistance-monitoring step (Lee et al. 2009, Clark 2009).

The bed bugs collected in 1993 appeared to be susceptible, as judged by $0 \%$ resistance allele frequencies at the two mutation sites (Table 3 ). In contrast, all the bed bugs collected in more recent years (20072009) appeared to be resistant to pyrethroids by possessing completely saturated or near-saturating levels of the L925I mutation and varying frequencies $(0-$ $100 \%$ ) of the V419L mutation. Based on this finding, it appears that the pyrethroid resistance allele, particularly the L925I mutation, has become more widespread in recent years. It was previously suggested that the V419L and L925I mutations do not always exist en bloc as a resistant haplotype (Yoon et al. 2008). The discovery of a bed bug population having only the L925I mutation confirms this notion and further suggests that there are at least two different Clvssc haplotypes, as follows: one having only the L925I mutation, and the other having both V419L and L925I mutations. Uniform distribution of the L925I mutation implies that it has been selected more intensively than the V419L mutation and plays a primary role in resistance to pyrethroids. Perhaps possession of L925I mutation may confer some advantage of fitness compared with insects possessing V419L mutation, which ultimately leads to its greater prevalence within the selected population.
As demonstrated in the current study, QS is particularly useful for genotyping on a population basis and in the prediction of resistance allele frequency in alcohol-stored bed bug specimens, thereby allowing the construction of a yearly regional database for resistance allele frequency. Once accumulated, this type of temporal and spatial resistance database will provide extremely valuable information for understanding the evolutionary process of resistance development in a region over time.

Along with QS, a FCVB protocol was established to determine pyrethroid resistance in field-collected bed bug populations. Based on the FCVB method, baseline susceptibility levels of bed bugs to deltamethrin and $\lambda$-cyhalothrin have been determined. Furthermore, resistance levels to these pyrethroids were determined to be $>6,990$-fold with limited laboratory selection. If this level of pyrethroid resistance is possible with such limited selection, it is imperative to curtail pyrethroid use immediately and to introduce alternative insecticides with different modes of action. Nevertheless, the V419L mutation is not yet uniform, as suggested from QS of field-collected bed bugs (Table 3 ), and supports the need for more intense resistance monitoring of bed bug field populations. In regions where the resistance allele frequencies are low or near zero, pyrethroids can be used cautiously under constant and precise resistance monitoring.

The procedures for bed bug capture from infested structures were simplified by employing a custommade aspirator that can be directly connected to the vial used in the FCVB. The battery-powered aspirator (such as the mini pump model MP-2N, Sibata, Japan) greatly facilitates the collection of any stage of bed bugs directly into the 5 -ml test vial. Simply placing an insecticide-treated filter paper strip into the vial containing captured bed bugs initiates the bioassay. At the 1-h discriminating concentration (164 and $174 \mathrm{ppm}$ for deltamethrin and $\lambda$-cyhalothrin, respectively) used in the FCVB (Table 2), the presence or absence of pyrethroid resistance can be determined very quickly and will facilitate on-site resistance detection. On-site monitoring of bed bug resistance based on the FCVB should allow rapid acquisition of information on resistance status, thereby enabling immediate management decision at the site of bed bug infestation.

In summary, both QS and the FCVB can be employed as DNA-based and bioassay-based resistancemonitoring tools, respectively, in a complementary manner for long-term management of bed bug resistance to pyrethroids. This approach will extend the effective lifetime of this safe and valuable group of insecticides.

\section{Acknowledgments}

We thank the Department of Public Works, U.S. Army Garrison-Yongsan, for its assistance in obtaining field-collected bed bugs, and the anonymous reviewers for their valuable comments and suggestions. 


\section{References Cited}

Blow, J. A., M. J. Turell, A. L. Silverman, and E. D. Walker. 2001. Stercorarial shedding and transtadial transmission of hepatitis B virus by common bed bugs (Hemiptera: Cimicidae). J. Med. Entomol. 38: 694-700.

Boase, C. J. 2001. Bed bugs: back from the brink. Pesticide Outlook. 12: 159-162.

Clark, J. M. 2009. Determination, mechanism and monitoring of knockdown resistance in permethrin-resistant human head lice, Pediculus humanus capitis. J. Asia-Pacific Entomol. 12: 1-7.

Day, J. F., F. D. Edman, S. E. Kunz, and S. K. Wikel. 2000. Direct injury: phobias, psychoses, annoyance, allergies, toxins, venoms and myiasis, pp. 99-149. In B. F. Eldridge and J. D. Edman (eds.), Medical entomology: a textbook on public health and veterinary problems caused by arthropods. Kluwer Academic Publishers, Dordrecht, The Netherlands.

Gangloff-Kaufmann, J., C. Hollingworth, J. Hahn, L. Hansen, B. Kard, and M. Waldvogel. 2006. Bed bugs in America: a pest management industry survey. Am. Entomol. 52: 105-106.

Jupp, P. G., and S. F. Lyons. 1987. Experimental assessment of bed bugs (Cimex lectularius and Cimex hemipterus) and mosquitoes (Aedes aegypti formosus) as vectors of human immunodeficiency virus. AIDS 1: 171-174.

Karunaratne, S.H.P.P., B. T. Damayanthi, M.H.J. Fareena, V. Imbuldeniya, and J. Hemingway. 2007. Insecticide resistance in the tropical bed bug Cimex hemipterus. Pestic. Biochem. Physiol. 88: 102-107.

Kwon, D. H., J. M. Clark, and S. H. Lee. 2004. Estimation of knockdown resistance in diamondback moth using realtime PASA. Pestic. Biochem. Physiol. 78: 39-48.
Kwon, D. H., K. S. Yoon, J. P. Strycharz, J. M. Clark, and S. H. Lee. 2008. Determination of permethrin resistance allele frequency of human head louse populations by quantitative sequencing. J. Med. Entomol. 45: 912-920.

Lee, S. H., J. M. Clark, Y. J. Ahn, W.-J. Lee, K. S. Yoon, D. H. Kwon, and K. M. Seong. 2009. Molecular mechanisms and monitoring of permethrin resistance in human head lice. Pestic. Biochem. Physiol. (in press).

Moore, D. J., and D. M. Miller. 2006. Laboratory evaluations of insecticide product efficacy for control of Cimex lectularius. J. Econ. Entomol. 99: 2080-2086.

Reinhardt, K., and M. T. Siva-Jothy. 2007. Biology of the bed bugs (Cimicidae). Annu. Rev. Entomol. 52: 351-374.

Romero, A., M. F. Potter, D. A. Potter, and K. F. Haynes. 2007. Insecticide resistance in the bed bug: a factor in the pest's sudden resurgence? J. Med. Entomol. 44: 175-178.

Scarupa, M. D., and A. Economides. 2006. Bed bug bites masquerading as urticaria. J. Allergy Clin. Immunol. 117: 1508-1509.

Ter Poorten, M. C., and N. S. Prose. 2005. The return of the common bed bug. Pediatr. Dermatol. 22: 183-187.

Usinger, R. L. 1966. Monograph of Cimicidae (Hemiptera, Heteroptera). Entomological Society of America, College Park, MD.

Xu, Q., H. Wang, L. Zhang, and N. Liu. 2006. Kdr allelic variation in pyrethroid resistant mosquitoes, Culex quinquefasciatus (S.). Biochem. Biophys. Res. Commun. 345: 774-780.

Yoon, K. S., D. H. Kwon, J. P. Strycharz, C. S. Hollingsworth, S. H. Lee, and J. M. Clark. 2008. Biochemical and molecular analysis of deltamethrin resistance in the common bed bug (Hemiptera: Cimicidae). J. Med. Entomol. 45: 1092-1101.

Received 16 November 2009; accepted 13 April 2010. 